\title{
Échos du centenaire de la Première Guerre mondiale sur Twitter
}

\section{Echoes of the Centenary on Twitter}

Frédéric Clavert, Université de Lausanne, Laboratoire de cultures et humanités digitales de l’Université de Lausanne (LADHUL).

\section{Résumé}

En nous fondant sur l'analyse d'un large corpus de tweets reliés à la Première Guerre mondiale d'une part, et des liens contenus dans ces tweets d'autre part, nous interrogeons la manière dont les activités des comptes Twitter marquent une appropriation du Centenaire. Nous formulons l'hypothèse que la base de données des « Morts pour la France » émerge en tant que « lieu de Mémoire ».

\begin{abstract}
Based on the analysis of a large corpus of tweets related to the First World War on the one hand and links contained in these tweets on the other hand, we question the ways activities of Twitter accounts show an appropriation of the Centenary. We hypothesize that the « Morts pour la France » database is currently emerging as a « Lieu de Mémoire ».
\end{abstract}

\section{Mots-clés}

Twitter, Centenaire, Première Guerre mondiale, Poilus, mémoire

\section{Keywords}

Twitter, Centenary, First World War, Fallen soldiers, memory 
Il est souvent rappelé, avec justesse, qu’à la différence des grands moments de commémoration de la Première Guerre mondiale au cours du $\mathrm{Xx}^{\mathrm{e}}$ siècle, son centenaire présente la particularité de se dérouler sans qu’il n’y ait plus aucun vétéran du conflit vivant. Une autre spécificité, de nature bien différente, est toutefois moins souvent évoquée : le Centenaire se déroule dans un contexte où les réseaux sociaux numériques occupent désormais une place importante dans le paysage médiatique. Bien que ces nouveaux médias aient largement été étudiés depuis leur naissance, au milieu des années 2000, peu d'enquêtes portent sur les traces de mémoire collective que l’on peut y déceler.

Pour comprendre l’impact des réseaux sociaux numériques sur la mémoire collective, nous nous concentrons ici sur Twitter $^{1}$, un réseau social numérique permettant à ses utilisateurs de publier sur le web des tweets ou, en français, des «gazouillis », c'est-à-dire de courts messages d'au maximum cent quarante caractères. À cette fonctionnalité centrale appelée micro-blogging, s'ajoute une dimension sociale : on peut suivre les messages de quelqu'un et, ainsi, les faire apparaître dans la page d'accueil de son compte sur Twitter. Cette fonctionnalité sociale, contrairement à un site comme Facebook, est asymétrique : quelqu’un que l'on suit n'est en effet pas obligé de vous suivre en retour. Twitter permet, grâce à ces fonctionnalités, une diffusion rapide de l'information et encourage le partage de contenu par l'inclusion, dans les tweets, de liens hypertexte pour compenser la faiblesse du nombre de caractères.

Twitter autorise un accès relativement facile à ses données. Ainsi, depuis avril 2014, avonsnous pu collecter un corpus de plus de trois millions de tweets liés à la Grande Guerre et à son centenaire ${ }^{2}$. Ces tweets contiennent tous un mot-clé relatif au conflit, souvent sous forme d'un hashtag (ou «mot-dièse »), c'est-à-dire un mot, une abréviation ou un sigle précédé d’un croisillon comme \#ww1 pour l'abréviation de World War One, ou \#pgm pour Première Guerre mondiale. Le mot-dièse est une fonctionnalité inventée par les utilisateurs de Twitter, 
pour donner une nuance ironique à un terme, insister sur un concept ou marquer sa volonté de participer à une discussion impliquant un réseau allant de quelques personnes à des centaines de milliers, comme dans le cas des tweets que nous avons collectés. Dans ces tweets figure souvent un hyperlien pointant vers une page web liée au centenaire.

L'intérêt d'un tel corpus contenant les traces d'un demi-million de comptes Twitter, est de permettre l'analyse des pratiques de ces comptes, dont la nature est toutefois variée et parfois difficile à cerner : particuliers, institutions de toute nature comme des musées ou des médias, projets de recherche ou encore bots, c'est-à-dire des comptes automatisés comme @RealTimeWW1, un compte mis en place par l’Université du Luxembourg dans le cadre d'un enseignement de master qui tweete automatiquement sur des sujets liés à la Grande Guerre à cent ans de distance. Ce corpus peut être étudié de différentes manières : nous pouvons y observer le contenu qui est publié et parfois discuté dans les tweets, dans deux langues principalement (anglais et français). Nous pouvons également les considérer comme un réseau ou un ensemble de réseaux et y reconstituer la circulation de l'information, en comparant éventuellement les deux aires linguistiques.

Comment les utilisateurs de Twitter se réapproprient-ils le centenaire de la Première Guerre mondiale ? Nous tenterons de répondre à cette question en deux temps : en exposant quelques conclusions générales issues de l'analyse de l'ensemble de notre corpus, puis en nous penchant plus précisément sur les tweets émis les 11 novembre 2014 et 2015, avec une insistance particulière sur les liens hypertextes partagés dans ces tweets le 11 novembre 2015.

\section{Le centenaire et Twitter : généralités}

Bien que certaines commémorations aient comporté une forte dimension internationale, notamment à Sarajevo en juin 2014, le centenaire de la Première Guerre mondiale a été 
surtout rythmé par des cadences nationales. Dans le cas français, les commémorations sont lancées officiellement en novembre 2013 et connaissent un certain nombre de temps forts au cours de l’année 2014 : assassinat de l'archiduc François-Ferdinand à Sarajevo, séquence sur les entrées en guerre à partir du 14 juillet, bataille de la Marne, et 11 novembre 2014. Si l’année 2015 est moins dense, les grandes batailles de 1916, Verdun et la Somme, ont été largement commémorées au cours de l'année 2016 et ont fait l’objet d'une coopération bilatérale, avec l'Allemagne pour la première, et le Royaume-Uni pour la seconde. Enfin, l’année 2018, centenaire de la sortie du conflit, devrait marquer elle aussi un temps fort des commémorations. Ce rythme plutôt national s’articule avec de nombreuses initiatives locales, la mémoire de la Première Guerre mondiale en France se structurant autant au niveau national que local $^{3}$. Cette imbrication entre local et national se retrouve au Royaume-Uni. Les commémorations y sont structurées autour de trois dates essentielles : le centenaire de l'entrée en guerre (4 août 2014) - dont certains éléments se prolongent jusqu'en novembre comme l'installation à la Tour de Londres de coquelicots en céramique symbolisant les soldats tombés au combat -, le déclenchement de la bataille de la Somme ( $1^{\mathrm{er}}$ juillet 2016 , avec la France), et la sortie de guerre. De nombreuses célébrations et initiatives locales ont pu compléter ce programme, faisant du centenaire la combinaison d'une «multitude de mémoires ${ }^{4}$. Retrouvons-nous ces rythmes sur Twitter? Comment s'articulent ces temporalités nationales et locales dans le corpus que nous avons collecté ?

Nous allons en premier lieu nous intéresser au nombre de tweets par jour, avant d'en analyser plus précisément la répartition linguistique entre français et anglais. Enfin, nous détaillerons quelque peu le contenu de ces tweets, en nous attachant aux différences entre le corpus anglophone et le corpus francophone. Notons que ne sont inclus dans la version du corpus étudiée ici que les tweets portant sur la période d’avril 2014 à avril 2016.

Le nombre de tweets (cf figure 1 : Nombre de tweets par jour) 
L’observation du nombre de tweets collectés par jour permet d'identifier les dates qui ont donné lieu au plus grand nombre de messages :

1. Centenaire de l'entrée en guerre du Royaume-Uni, 4 août 2014 : 110684 tweets ;

2. Commémoration de l’armistice, 11 novembre 2014 : 91108 tweets ;

3. Commémoration de l'armistice, 11 novembre 2015 : 73520 tweets ;

4. Centenaire du déclenchement de la guerre de Verdun, 21 février 2016 : 25011 tweets ;

5. Centenaire de l'assassinat de l'archiduc François-Ferdinand, 28 juin 2014 : 12029 tweets ;

6. Easter Rising, 28 et 29 mars 2016 : 12021 et 13299 tweets respectivement.

7. ANZAC Day, 25 avril : 7605 tweets.

Le centenaire du déclenchement de la bataille de la Somme, le $1^{\text {er }}$ juillet 2016, n’est pas inclus ici alors que c'est à cette date que l'on constate la plus forte activité sur Twitter en relation avec la Première Guerre mondiale. Il manque aussi la commémoration franco-allemande de la bataille de Verdun en mai 2016, qui a donné lieu à d’intenses polémiques. Si nous nous concentrons sur la période allant d'avril 2014 à avril 2016, l’événement le plus marquant fut bien le centenaire des entrées en guerre et en premier lieu celui de l'entrée en guerre du Royaume-Uni. Viennent ensuite les 11 novembre 2014 et 2015, seul moment récurrent dans le corpus étudié. Les autres dates importantes sont le centenaire de l'assassinat de l'archiduc François-Ferdinand, événement emblématique dans le déclenchement de la Grande Guerre ; l’ANZAC Day qui célèbre le débarquement allié à Gallipoli avec des troupes venant essentiellement d'Australie et de Nouvelle Zélande et qui est devenu le jour annuel consacré aux anciens combattants dans ces deux pays ${ }^{5}$; le centenaire du déclenchement de la guerre de la bataille de Verdun en février 2016 ; enfin, le centenaire de l’Easter Rising, à la fois moment-clé de la Première Guerre mondiale et événement crucial pour la marche vers l’indépendance irlandaise. 
La répartition linguistique du corpus (cf Figure 2 - Temporalités et répartition linguistiques du corpus. Répartition entre les langues française et anglaise. Dans cette figure, plus le gris clair est présent, plus le nombre de tweets anglophones est important. À l'inverse, plus le gris foncé est visible, plus nombreux ont été les tweets francophones ce jour là).

La répartition linguistique permet d’affiner notre première analyse. Rappelons en premier lieu que le système de collecte que nous avons mis en place se fonde sur des mots-clés : si un tweet comprend l'un des mots-clés que nous avons sélectionnés, il est inclus dans notre base de données. Parmi les mots-clés collectés, figurent des termes en anglais, français et allemand. Or, le nombre de tweets en allemand n'est pas suffisant pour apparaître sur le graphique (figure 2). Les traces de mémoire de la Première Guerre mondiale en Allemagne sont ainsi trop faibles et confirment que la Grande Guerre, pour diverses raisons, n'est pas un objet de mémoire outre-Rhin aussi fort qu’il ne l'est en France ${ }^{6}$. Trois des événements les plus twittés des commémorations sont majoritairement francophones : les deux 11 novembre et l’ouverture des commémorations de la bataille de Verdun en février 2016. Le centenaire des entrées en guerre est par contre largement anglo-saxon, avec en particulier le 4 août 2014 pour le Royaume-Uni. Nous retrouvons ainsi sur Twitter le rythme différencié des commémorations de part et d'autre de la Manche. En outre, que l'entrée en guerre soit une date plus marquante en Grande Bretagne rappelle que la participation du Royaume-Uni à la Première Guerre mondiale n'était pas une évidence et a été contestée jusque dans l'aprèsguerre. Enfin, l'ANZAC Day et le centenaire de l'Easter Rising sont très logiquement eux aussi majoritairement anglophones.

Le contenu des tweets : divergences et points communs

Lorsque l'on analyse le contenu des tweets dans les deux corpus, anglophone et francophone, il est intéressant de mesurer à la fois les divergences et les points communs. La première similitude touche aux omissions : si les deux corpus parlent des soldats morts sur le champ de 
bataille de manière différente, l’absence ou quasi-absence des femmes, des troupes coloniales, des fusillés - qui pourtant font l'objet d'une base de données sur « Mémoire des Hommes ${ }^{7}$ » et, dans le cas de la France et de la Belgique, des habitants des territoires occupés, se retrouve dans les deux corpus. Le front de l'Est, les relations internationales, les causes et conséquences de la Grande Guerre sont également peu évoqués. Le second point commun est le pendant de ces absences, c'est-à-dire la focalisation sur les soldats morts au front. Le troisième est l’importance donnée aux combats pour rythmer ces traces de mémoire.

Ces deux derniers points font apparaître également des divergences. L’hommage aux Poilus est central côté francophone, alors que, côté britannique, l'évocation des soldats se fait au travers de la mémoire des champs de bataille. Les autorités du Royaume-Uni ayant pris au lendemain de la guerre la décision de ne pas rapatrier les corps des soldats tombés sur les champs de bataille, la focalisation autour de ces derniers semble aller de soi. Concernant les batailles, si Verdun constitue un événement fondamental dans le corpus francophone, Ypres et la Somme sont nettement plus présentes dans les tweets anglophones. Cette hiérarchisation apparente des batailles sera toutefois peut-être modifiée lorsque nous aurons inclus dans l’étude les tweets émis pendant les commémorations de la bataille de la Somme.

La focalisation du corpus francophone autour de la figure des Poilus fait de lui un ensemble que l’on pourrait qualifier de «mémoriel ». Toutefois, cela ne signifie pas que le souvenir des Poilus s’exprime de manière analogue tout au long de l'année. Si, à l'occasion du 11 novembre ou d'autres commémorations officielles comme celle du déclenchement de la bataille de Verdun, le vocabulaire qui domine le contenu des tweets se rattache à un champ lexical touchant à l’hommage rendu de manière très générale aux Poilus, les soldats français sont en revanche évoqués différemment le reste de l’année: sont alors décrits plus précisément les parcours individuels des Poilus. Cette différence s’explique par le recours à la 
base de données des soldats « Morts pour la France ${ }^{8}$ » sur le site « Mémoire des hommes » et par l’activité liée à son indexation.

Les grandes commémorations auraient-elles par conséquent un effet sur les interactions autour du centenaire sur Twitter ? Si elles provoquent une augmentation très importante de l’activité, avec des échanges autour de contenus liés directement à la commémoration en cours, elles n’empêchent pas la plupart du temps les activités de se poursuivre sur le reste de l'année sur un mode plus discret. Peut-être voit-on ici aussi l'un des effets de l'articulation entre les commémorations organisées par l’État et le fait que la mémoire collective soit par ailleurs prise en charge par un ensemble d'acteurs non-étatiques : des collectivités territoriales et des groupes de particuliers, ces derniers étant actifs toute l'année et pas seulement au moment des grandes cérémonies liées au calendrier officiel des commémorations.

Le dernier grand contraste repéré entre les deux aires linguistiques ne tient pas tant au contenu qu'à la qualité des comptes Twitter qui émettent les tweets. Twitter propose des fonctionnalités sociales qui favorisent la circulation des informations en permettant de suivre d'autres comptes Twitter ou d'être suivi par d'autres comptes; de «retweeter» le « gazouilli » d'un autre compte, c'est-à-dire citer un tweet, soit sans aucune modification, soit avec un commentaire ; et, enfin, de mentionner dans un tweet un autre compte Twitter. Il est par conséquent nécessaire de regarder qui suit ou est suivi, et de s’intéresser au nombre et à la nature de ces liens.

Si l'on analyse les retweets et les mentions présentes dans notre corpus, on observe un groupe francophone très centré autour du compte Twitter de la Mission du centenaire ${ }^{9}$, qui est chargée de coordonner les commémorations en France. La structure des retweets et mentions côté anglophone, quant à elle, est plus diverse et moins centralisée. Les comptes de la BBC, des Imperial War Museums, ou encore des anciens combattants (PoppyLegion), sont certes très actifs, mais les comptes liés à des projets d' « histoire publique » jouent eux aussi un rôle 
important. Cette discipline, davantage présente aux États-Unis et au Royaume-Uni qu’en France, est une forme d’histoire « appliquée » qui insiste sur les relations entre les citoyens et leur histoire et favorise souvent l'appropriation de la seconde par les premiers.

Cette différence entre les groupes francophone et anglophone appelle plusieurs remarques. Elle reflète tout d'abord des approches différentes du centenaire, avec une nette centralisation en termes de communication, même s’il faut la relativiser, côté français. Elle montre aussi que les musées et, de manière plus large, les institutions « mémorielles » en France ne suivent pas les usages de Twitter, comme utiliser un «hashtag» pour rejoindre une discussion plus globale sur la Grande Guerre. Certains musées semblent plus à l'aise pour leur communication numérique avec d'autres outils, tels que Facebook, un réseau qui facilite peutêtre davantage la maîtrise des interactions entre l'institution et les internautes. En témoigne le succès du Poilu fictif, Léon Vivien, mis en scène sur une page Facebook par le Musée de la Grande Guerre de Meaux. Cet exemple reste toutefois original dans le paysage muséal français ${ }^{10}$.

Si nous avons jusqu'ici essayé d’analyser le contenu des tweets, il est une spécificité que nous n’avons pas encore abordée : Twitter, en imposant des messages courts, favorise la diffusion d'hyperliens vers des pages web qui contiennent une information ou un argumentaire plus détaillés que la teneur d’un message limité à cent quarante caractères. Quel est alors le contenu des liens ainsi communiqués ? Nous allons aborder cette question à travers le cas francophone du 11 novembre.

\section{Les partages de liens : le cas des 11 novembre 2014 et 2015}

Comme nous l'avons rappelé plus haut, le 11 novembre est un jour particulier en France, pierre angulaire des commémorations de la Première Guerre mondiale, devenu «jour du 
Souvenir » dès 1922. Le 11 novembre 2013 a marqué le lancement officiel des commémorations du centenaire et le 11 novembre 2014 l'un de ses points d'orgue, avant Verdun et la Somme en 2016. Comme l'analyse quantitative des tweets le démontre ( $c f$ Figures 1 et 2), les 11 novembre 2014 et 2015 sont, avec le 21 février 2016, les dates où les tweets francophones sont majoritaires.

L'étude du contenu des tweets en français des 11 novembre montre que différents sujets sont abordés avec notamment la nature fériée du 11 novembre, ou bien les lieux et événements propres au 11 novembre de l'année en cours comme la présence de François Hollande en 2014 sur le site de la nécropole Notre-Dame-de-Lorette (Pas-de-Calais). Mais la thématique la plus importante est bien celle liée aux Poilus : le 11 novembre est ainsi sur Twitter, et ailleurs, un jour d'hommage aux soldats « Morts pour la France ». Depuis 2012, il est d'ailleurs devenu officiellement un jour d'hommage à tous les soldats morts en opération. Les liens qui ont été communiqués dans ces tweets francophones ont-ils le même type de contenu ? Nous allons ici nous pencher sur le cas du 11 novembre 2015.

Descriptif du corpus de pages web du 11 novembre 2015

Le 11 novembre 2015, 73520 tweets liés à la Première Guerre mondiale, majoritairement francophones, ont été stockés dans notre base de données. Ils contenaient 26067 liens en comptant tweets et retweets. Si l'on se contente des tweets originaux en retirant les retweets, nous avons comptabilisé 9818 liens pour 21046 tweets. Après un dédoublonnage et en omettant les liens pointant vers du matériel non textuel, nous disposons d’un corpus de 3600 liens environ, soit un peu plus de 1000 pages web si l'on se limite aux textes en français. Pour analyser ce corpus, nous allons nous attarder sur les liens et les sites web les plus « populaires », sur les comptes qui les ont publiés sur Twitter et sur les grands thèmes abordés à travers leur contenu.

Les liens les plus partagés 
La plupart des pages web dont le lien a été tweeté le 11 novembre 2015 l’ont été une seule fois. Le lien le plus tweeté l'a été quatre cents fois (hors retweet). Si nous regardons les domaines des liens - c'est-à-dire non la page précise mais le site web dans lequel elle se situe - nous constatons que la base de données des « Morts pour la France » apparaît plus de deux cents cinquante fois ( $c f$ Erreur ! Source du renvoi introuvable.-Domaines les plus tweetés). Elle est plus souvent tweetée que des liens pointant vers des plateformes majeures du web comme Twitter, Facebook, Instagram, Mashable, ou encore Daily Motion qui sont, pour ce type d'analyse et de corpus, indépendamment du sujet abordé, toujours parmi les plus tweetées. Viennent ensuite des médias (France 3 régions, Le Monde, L’Yonne, Ouest France, France Bleu et La Voix du Nord), dont un seul, Le Monde, est national. La présence de plombiers-paris-75.com est liée à une forme de «spam»: une entreprise profite d'un événement populaire pour faire de la publicité, phénomène que l’on retrouve souvent sur les réseaux sociaux numériques. Enfin, beaucoup de liens sont dirigés vers le site de la Mission du centenaire.

\section{Qui tweete des URLs?}

Les échanges de ces URLs entre utilisateurs de Twitter restent dominés par des médias relativement traditionnels. Le compte de la chaîne de télévision généraliste France 2, @France2TV, qui a retransmis la cérémonie et tweeté à son sujet, fournit par exemple des liens amenant un internaute vers le site de la chaîne ou vers les sites d’autres médias. Les comptes des autres médias actifs font de même. Dans l'ensemble, ces comptes « médiatiques » ne renvoient pas ou peu vers la base de données des « Morts pour la France ». Ce niveau d'échange de liens, très visible, en masque un autre, constitué de comptes de particuliers, où cette fois-ci, les liens partagés renvoient souvent vers la base de données des « Morts pour la France ». Parmi ces comptes Twitter, beaucoup sont reliés au défi « Un jour un poilu ${ }^{11}$ », initiative du compte Twitter @1j1p. Ce groupe utilise le module d'indexation de 
la base de données des « Morts pour la France » afin de transcrire les fiches, ce qui permettra, à terme, de transformer cette base en un outil de recherche - universitaire, généalogique ou autre - très performant.

L'étude des liens tweetés le 11 novembre 2015 montre donc deux niveaux de partage : un niveau très visible, constitué de comptes Twitter de grands médias, et un niveau invisible, un phénomène « infra », qui est marqué par l'importance du site Mémoire des Hommes et de sa base de données des soldats « Morts pour la France ». Ce niveau moins visible est constitué de comptes de particuliers, dont le groupe qui s’est formé autour de l’initiative « 1 jour - 1 poilu ».

Le contenu francophone des pages web partagées

Quel est le contenu de ces pages web dont le lien est partagé (cf Figure 4 - Principaux thèmes évoqués dans les pages dont les liens ont été tweetés le 11 novembre 2015) ? La représentation graphique des principaux thèmes évoqués est obtenue par un traitement informatique du corpus de pages web qui revient à transformer du texte en matière statistique en se fondant sur la cooccurrence des mots. Le logiciel que nous utilisons regroupe des segments de textes contenus dans ces pages web ayant des caractéristiques similaires à l’intérieur des classes. Chaque classe correspond à un grand thème évoqué dans le corpus. Les mots mis en évidence sous forme de nuages sont les mots les plus significatifs de ces classes.

Beaucoup de pages web évoquent les caractéristiques du 11 novembre 2015 (cf Figure 4-1). La cérémonie principale se déroulait sous l'Arc de Triomphe, au pied de la flamme du Soldat inconnu. François Hollande, président de la République, y avait invité son prédécesseur, Nicolas Sarkozy. Certains sujets politiques - notamment en lien avec cette invitation - sont évoqués (cf Figure 4-2). Si les termes typiques de l’hommage rendu à l'occasion du 11 novembre sont bien présents ( $c f$ Figure 4-8), nous trouvons également des éléments plus historiques ( $c f$ Figure 4-3, 5, 4) ainsi que les catégories liées à l'indexation de la base de 
données des « Morts pour la France » (cf Figure 4-7), indexation qui donne aussi l’occasion de parler des familles et du parcours des soldats ( $c f$ Figure 4-9). Enfin, une catégorie difficile à interpréter est celle où l'on observe le vocabulaire lié à l'écriture : on y retrouve notamment des extraits de lettres de Poilus.

En reliant ces catégories de discours au «poids » des pages - nous définissons ici le «poids » des pages comme le nombre de fois où le lien a été cité ou retweeté -, nous constatons que les catégories de discours correspondant aux tweets les plus cités sont les catégories (1) et (8), c’est-à-dire les catégories classiques du 11 novembre. Mais celles qui sont relatives aux Poilus, à leur famille, à l'histoire, correspondent à des tweets nettement moins diffusés, mais bien plus nombreux.

Ainsi, un internaute peu averti qui a consulté Twitter le 11 novembre 2015 a pu observer essentiellement les tweets des grands médias classiques, contenant surtout des liens vers leurs propres pages web. Il n’a probablement pas pu voir des éléments bien moins visibles, mais beaucoup plus riches, relatifs aux Poilus et avec en position centrale la base de données des « Morts pour la France ».

Il existe une forte différence entre la masse très visible d'information sur les cérémonies du 11 novembre et celle, moins visible, qui comprend des renseignements très détaillés sur les Poilus et leur parcours. Ce deuxième ensemble, comme nous l'avons expliqué en première partie, est par ailleurs bien présent sur Twitter tout au long de l'année, et la base de données des « Morts pour la France » en constitue le point focal.

En conséquence, pour comprendre la manière dont les membres du réseau Twitter se réapproprient le centenaire de la Première Guerre mondiale, il faut s’arrêter sur le statut de 
cette base de données. Notre hypothèse est qu'aujourd’hui, au-delà d’un outil de recherche, cette base de données est devenue un véritable « lieu de mémoire » en ligne.

La définition de cette notion s’est révélée très malléable, depuis la parution dans les années 1980 des travaux dirigés par Pierre Nora. Si nous nous référons à la définition proposée par M. Margue ${ }^{12}$, cette notion insiste sur le rôle de l'histoire collective, avec d'une part un triptyque "symbole, transmission, intégration », et d'autre part, une double opposition « mémoire / oubli » et «minorité / majorité ». Ces critères nous paraissent bien s’appliquer à la base de données des «Morts pour la France» lorsque l'on analyse les tweets qui l'évoquent : ainsi, le discours du défi « 1 jour - 1 poilu » revendique ouvertement la volonté de transmettre une mémoire et de rendre hommage à des soldats qui se sont sacrifiés pour une cause plus grande qu’eux. Indexer la base de données des « Morts pour la France » est alors perçu comme le fruit d'une volonté de lutter contre l'oubli de ce sacrifice et un moyen d'en entretenir la mémoire ${ }^{13}$. Cette polarisation autour de la figure du Poilu - qui va bien au-delà de ce défi - risque toutefois de rendre cette mémoire très sélective, notamment en ignorant les autres expériences de la guerre, y compris combattantes (prisonniers et fusillés).

Dans ce cadre, il nous semble donc que l'on peut percevoir la base de données des « Morts pour la France » comme une sorte de «lieu de mémoire » en ligne : de nombreux comptes Twitter évoquent cette base comme un outil de lutte contre l'oubli par l'hommage rendu aux soldats. La base de données devient, progressivement, un « lieu de mémoire par le bas », dans le sens où les grands organes médiatiques présents sur Twitter en parlent peu, quand les particuliers, passionnés de la Première Guerre mondiale ou simplement intéressés par le passé de leur famille, font de la base le centre de leur activité de mémoire. La base de données des « Morts pour la France » est ainsi devenue un lieu de réappropriation de la mémoire de la Première Guerre mondiale, même si cette réappropriation ne passe pas par un questionnement de cette mémoire, qui reste classiquement centrée autour de la figure du Poilu. 
$1<$ http://www.twitter.com/>.

2 Pour les détails techniques de la collecte et de l'analyse de notre corpus: Frédéric Clavert, "Les commémorations du Centenaire de la Première Guerre mondiale sur Twitter”, Ricerche Storiche, a, vol. XLVI, $\mathrm{n}^{\circ}$ 2, 2016, à paraître.

${ }^{3}$ Romain Fathi, “French commemoration : The centenary effect and the (re)discovery of 14-18”, Australian Journal of Political Science vol. 50, n 3, juillet 2015, p. 545-552.

${ }^{4}$ Keith Jeffery, “Commemoration in the United Kingdom : A multitude of memories”, Australian Journal of Political Science vol. 50, n 3, juillet 2015, p. 562-567.

${ }^{5}$ Australian and New Zealand Army Corps (ANZAC) : le corps d'armée australien et néo-zélandais. L’ANZAC Day, le 25 avril, commémore la sanglante bataille de Gallipoli entre l'ANZAC et l'armée ottomane en 1915.

${ }^{6}$ Pour plus de détails sur la mémoire de la Grande Guerre en Allemagne : Nicolas Patin, “La Grande Guerre : un angle mort de l'histoire allemande ?", Histoire@Politique, n² 22, octobre 2014, p. 50-68.

${ }^{7}<$ http://www.memoiredeshommes.sga.defense.gouv.fr/fr/article.php?larub=3\&titre=premiere-guerre-mondiale $>$. Le site «Mémoire des hommes » crée par le ministère de la Défense comporte une partie très riche sur la Première Guerre mondiale: fiches des soldats «morts pour la France », base de données sur les fusillés, historiques régimentaires des unités militaires engagées dans la Grande Guerre.

$8<$ http://www.memoiredeshommes.sga.defense.gouv.fr/fr/article.php?larub=24\&titre=morts-pour-la-france-dela-premiere-guerre-mondiale $>$.

${ }^{9}<$ https://twitter.com/mission1418?lang=fr $>$.

$10<$ https://www.facebook.com/leon1914/>. Pour une analyse plus précise de cette page : Céline Schall, JeanChristophe Vilatte, “Le quotidien d'un poilu de la Première Guerre mondiale sur Facebook : nouvelle forme de médiation, nouvelle expérience ?”, in Cécile Camart, François Mairesse, Cécile Prévost-Thomas (dir.), Les mondes de la médiation culturelle, volume 2, Paris, L’Harmattan (Les cahiers de la médiation culturelle), 2016, p. 57-70.

${ }^{11}<$ https://www.1jour1poilu.com/hommage_poilus_grande_guerre_11_jours_11_novembre.php>. Jean-Michel Gilot est à l'origine de ce défi « 1 jour -1 poilu, 11 jours pour le 11 novembre » lancé depuis son compte Twitter pour « inviter les internautes à rendre hommage aux victimes en transcrivant des fiches individuelles permettant de les recenser, de mieux les connaître (à partir de leur commune d'origine, de leur grade, de leur régiment) et de préciser les circonstances de leur décès ".

${ }^{12}$ Michel Margue, “Lieux de mémoire au Luxembourg, lieux de mémoire en Europe”, in Benoît Majerus, Sonja Kmec, Michel Margue, Pit Péporté (dir.), Dépasser le cadre national des «Lieux de mémoire »: innovations méthodologiques, approches comparatives, lectures transnationales, Bruxelles, PIE-Peter Lang, 2009, p. 7-22.

${ }^{13}$ Voir l'interview de Jean-Michel Gilot par le site web En Envor, « 5 questions à un labellisé », non daté. En ligne : <http://enenvor.fr/eeo_actu/5_questions_a/5_questions_a_un_labellise.html>. Consulté le 18 septembre 2016. En outre, on pourra se reporter au site web du défi : <https://www.1jour1poilu.com/>. 